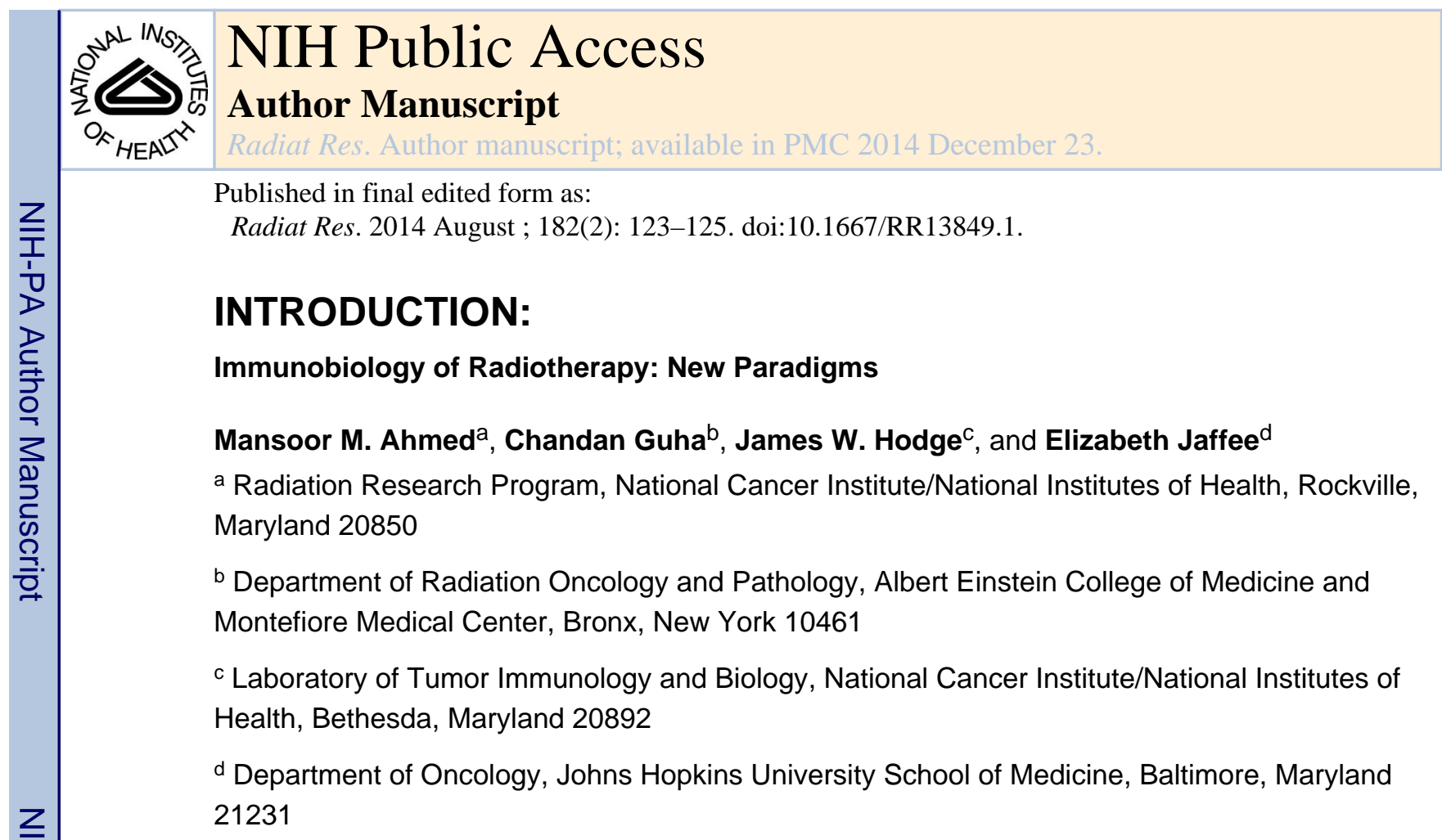

It has been well demonstrated that irradiated dying cancer cells release tumor antigens. The extracellular antigens and dying tumor cells are engulfed by circulating bone marrowderived antigen-presenting cells (APCs). After antigen uptake, APCs migrate to lymph nodes, where they engage with helper T cells for post-stimulation and APC activation. Induction of Th1 response and the activation of APCs further stimulate the induction of tumor specific cytotoxic T lymphocytes (CTLs) that could potentially clear tumor cells both at primary and metastatic sites (Fig. 1).

Radiation-induced immune modulation happens in two important phases. First, radiation induces damage-associated molecular pattern (DAMP) molecules. In this event, radiation normalizes tumor vasculature, modulates tumor cell phenotype and increases immune recognition of the tumor cell. Radiation treatment can cause: a. upregulation of chemokines and adhesion molecules, providing signals for $\mathrm{T}$ cells to be attracted to the tumor; and $\mathrm{b}$. upregulation of MHC molecules and tumor-associated antigens, making it easier for endogenous or immunotherapy-induced T cells to recognize and kill tumor (immunogenic modulation). Second, amplification by abrogating immune checkpoint factors with simultaneous costimulation of effector factors can ultimately lead to the induction of multiple unique T-cell populations (antigen cascade) that can kill antigen disparate tumor cells at metastatic sites (systemic effect) (Fig. 2).

\title{
Radiation-Induced Immunomodulation
}

This issue highlights novel findings and concepts on the immunobiology of radiation therapy coupled with translational concepts. Wattenberg et al. (1) reported on several cases where radiation modulates tumor cells to undergo immunogenic cell death or immunogenic modulation and this immune response is directly proportional to radiation dose. Current clinical radiotherapy regimens involve both hypo- and hyperfractionated treatments.

Therefore, it is important to understand how immune genes respond to survival adaptation of irradiated tumor cells (during multifractionation as well as after single high-dose fraction) to 
best exploit radiation for combined immunotherapy approaches. Aryankalayil et al. (2) demonstrated that both multifractionated as well single-dose radiation induced DAMPs and positively modulated the cytokine environment, with more observed in multifractionated radiotherapy. Kanagavelu et al. (3) demonstrated the presence of immune modulation in partially irradiated contralateral syngeneic lung tumors. Interestingly, partial tumor volume irradiation of the primary tumor led to an increased presence of infiltrating lymphocytes in both primary and distant tumors, unlike total tumor volume irradiation, suggesting that there is depletion of $\mathrm{T}$ cells in total tumor volume high-dose irradiation. To interrogate T-cell sensitivity, Filatenkov et al. (4) demonstrated that autologous T-cell infusion with high-dose hypofractionated radiation could compensate the lymphodepletive conditions for a more effective outcome than high-dose hypofractionated radiotherapy alone. These findings emphasize the need to combine radiation therapy with immune augmenting strategies for better outcomes. Along similar lines, there is an argument that radiation therapy alone does not have the intensity to generate enough proinflammatory signals to help overcome radiation-induced immune suppressive factors (5). Therefore, a partnership of radiation with targeted immunotherapy agents appears to be important to achieve therapeutically effective antitumor immune response and this partnership has shown some promising outcomes in preclinical and clinical settings (5).

\section{Myeloid Cells in Radiation-Induced Immunomodulation}

Macrophages and dendritic cells (DCs) play important roles in radiation-induced immune modulation. Adaptive immune response triggered by radiation therapy for tumor control can be negated by several factors activated through tumor myeloid cells ( 6 ). One such factor is Arginase I, which was shown to have a role in improving radiation-treated tumor control, although targeting multiple pathways in macrophage differentiation maybe a more effective tumor control strategy (6). Dendritic cells are important for engulfment of dying tumor cells during the radiation-induced immunomodulation process. Flt3L is a potent cytokine for stimulating tumor-specific responses and inducing proliferation of DCs. Kawashita et al. (7) demonstrated the efficacy of hepatocellular tumor control treated with radiation-inducible suicide gene therapy combined with Ad-Flt3L transfer. CD40L binds to CD40 on DCs to induce activation and maturation of DCs for tumor-specific response (7). In a followup study, Kawashita et al. (8) demonstrated an augmented antitumor immune response when CD40L was combined with radiation-inducible gene therapy plus Adeno-Flt3L in an orthotopic tumor model of hepatocellular carcinoma (8). Dendritic cell-based immunotherapy has shown promise in combination settings. The activation of effector DCs through radiation-induced Toll-like receptor (TLR) signaling presents a strong rationale for the potential to integrate radiotherapy with DC-based immunotherapy strategies (9).

\section{Radiation-Induced Immunomodulation and Antigen Presentation}

Crosstalk between immunomodulation and signal transduction pathways is vital when considering the use of targeted immune therapy. Verbrugge et al. (10) reported on the influence of crosstalk, and demonstrated that mTOR activity is important for achieving the efficacy of radio-immunotherapy. 


\section{Immune Therapeutics in Radiation Therapy}

Recently, there has been an increase in preclinical and clinical trials with new immune agents that target the inhibition of immune checkpoint proteins and thereby augment coimmune stimulatory proteins. In this era of modern immune therapeutics, it is important to understand the biologics, for example, Almo and Guha (11) examined the parameters of how affinity, selectivity, oligomeric state and valency can play major roles when designing studies that include radiotherapy.

Immunotherapy has shown efficacy in melanoma and renal cell carcinoma, however, its role in ovarian cancer is still under investigation despite evidence that it is an immunogenic disease. Although radiotherapy is not a mainstream regimen for ovarian cancer, data suggest that it should nevertheless be investigated as a means to exploit the high immunogenicity phenotype of this disease (12).

It is evident from the literature as well as the recent trends reported in this focus issue that radiation therapy as part of immunotherapy represents an attractive combination for clinical trials. Sharon et al. (13) provides important challenges such as preclinical design, dose/dose fractionation, overlapping toxicities, efficacy end points and statistical approaches that should be carefully addressed in the design of clinical trials.

Immunobiology of radiation therapy could contribute significantly to the treatment of aggressive solid tumors. To help advance the field, this focus issue provides scientific thinking in various facets of immuno-radiobiology, which has the potential for rapid clinical translation into novel cancer therapeutics.

\section{REFERENCES}

1. Wattenberg MM, Fahim A, Ahmed MM, Hodge JW. Unlocking the combination: potentiation of radiation-induced antitumor responses with immunotherapy. Radiat Res. 2014; 182:126-138. [PubMed: 24960415]

2. Aryankalayil MJ, Makinde AY, Gameiro SR, Hodge JW, Rivera-Solis PP, Palayoor ST, et al. Defining molecular signature of pro-immunogenic radiotherapy targets in human prostate cancer cells. Radiat Res. 2014; 182:139-148. [PubMed: 25003313]

3. Kanagavelu S, Gupta S, Wu X, Philip S, Wattenberg MM, Hodge JW, et al. In vivo effects of lattice radiation therapy on local and distant lung cancer: potential role of immunomodulation. Radiat Res. 2014; 182:149-162. [PubMed: 25036982]

4. Filatenkov A, Baker J, Muüller AM, Ahn G-O, Kohrt H, Dutt S, et al. Treatment of 4T1 metastatic breast cancer with combined hypofractionated irradiation and autologous T-cell infusion. Radiat Res. 2014; 182:163-169. [PubMed: 24992165]

5. Demaria S, Pilones KA, Vanpouille-Box C, Golden E, Formenti SC. The optimal partnership of radiation and immunotherapy: from preclinical studies to clinical translation. Radiat Res. 2014; 182:170-181. [PubMed: 24937779]

6. Crittenden MR, Savage T, Cottam B, Baird J, Rodriguez PC, Newell P, et al. Expression of Arginase I in myeloid cells limits control of residual disease after radiation therapy of tumors in mice. Radiat Res. 2014; 182:182-190. [PubMed: 24992164]

7. Kawashita Y, Deb NJ, Garg M, Kabarriti R, Alfieri A, Takahashi M, et al. An autologous in situ tumor vaccination approach for hepatocellular carcinoma. 1. Flt3 ligand gene transfer increases antitumor effects of a radio-inducible suicide gene therapy in an ectopic tumor model. Radiat Res. 2014; 182:191-200. [PubMed: 24972258] 
8. Kawashita Y, Deb NJ, Garg MK, Kabarriti R, Fan Z, Alfieri AA, et al. An autologous in situ tumor vaccination approach for hepatocellular carcinoma. 2 . Tumor-specific immunity and cure after radio-inducible suicide gene therapy and systemic CD40-ligand and Flt3-ligand gene therapy in an orthotopic tumor model. Radiat Res. 2014; 182:201-210. [PubMed: 24992166]

9. Roses RE, Datta J, Czerniecki BJ. Radiation as immunomodulator: implications for dendritic cellbased immunotherapy. Radiat Res. 2014; 182:211-218. [PubMed: 24992163]

10. Verbrugge I, Gasparini A, Haynes NM, Hagekyriakou J, Galli M, Stewart TJ, et al. The curative outcome of radioimmunotherapy in a mouse breast cancer model relies on mTOR signaling. Radiat Res. 2014; 182:219-229. [PubMed: 24960417]

11. Almo SC, Guha C. Considerations for combined immune checkpoint modulation and radiation treatment. Radiat Res. 2014; 182:230-238. [PubMed: 25003312]

12. Zakharia Y, Rahma O, Khleif SN. Ovarian cancer from an immune perspective. Radiat Res. 2014; 182:239-251. [PubMed: 25036981]

13. Sharon E, Polley M-Y, Bernstein MB, Ahmed M. Immunotherapy and radiation therapy: considerations for successfully combining radiation into the paradigm of immuno-oncology drug development. Radiat Res. 2014; 182:252-257. [PubMed: 25003314] 


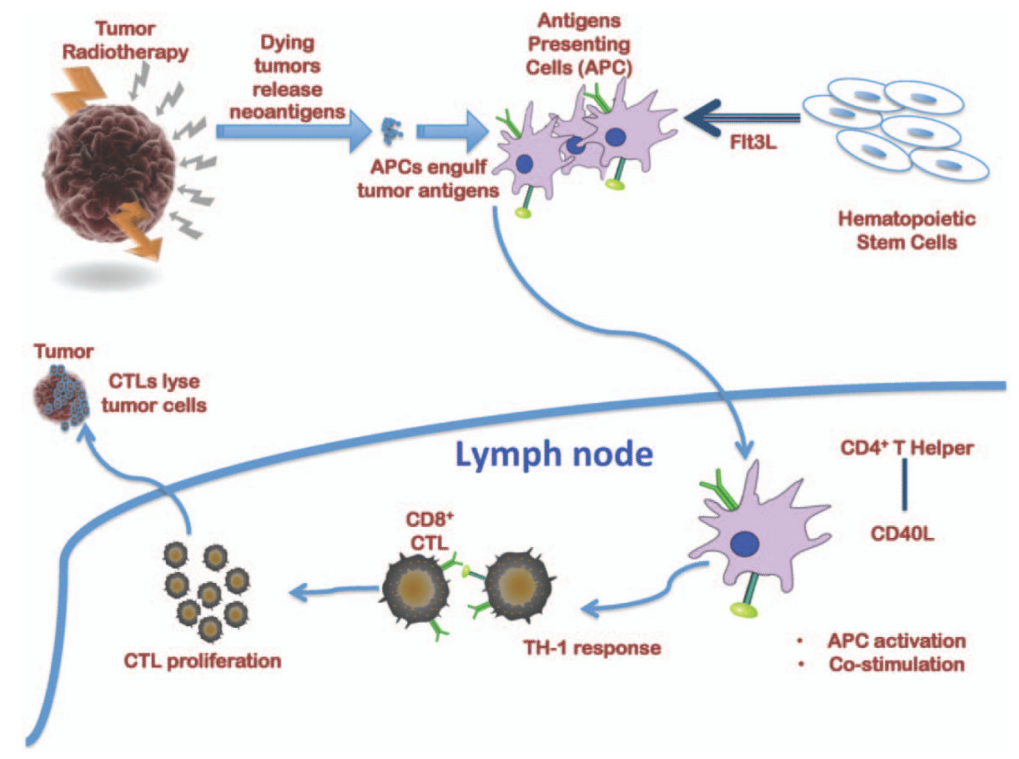

FIG. 1.

Schematic representation of the envisioned mechanism of radiation-primed immunotherapy of advanced cancer. 


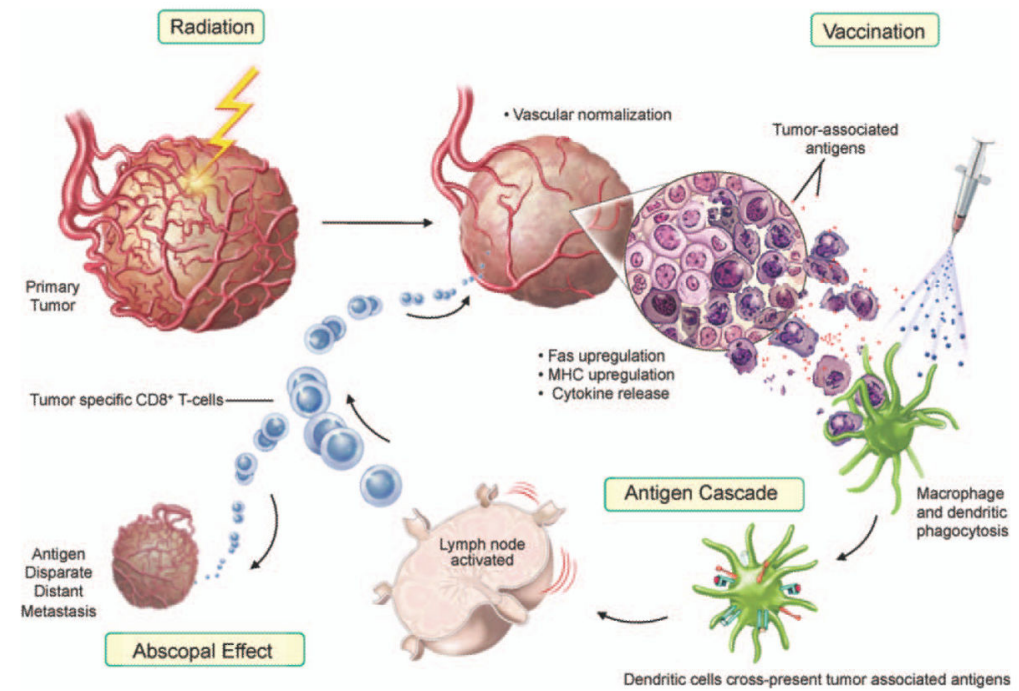

FIG. 2.

Model for radiation-induced immunogenic modulation. 